Rev. salud pública. 9 (4):506-515, 2007

\title{
Dança Folclórica e Caminhada: Um Estudo Comparativo do Gasto Calórico de Universitários
}

\section{Folk-dancing and walking: a comparative study of college students' calorific output}

Solange Maria Ribeiro-Nunes ${ }^{1}$, Aurinice Sampaio Irene-Monte ${ }^{1}$, Rogério FerreiraEmygdio $^{2}$ e Maria Irany Knackfuss ${ }^{3}$

1 Universidade Estadual do Piauí-Brasil solangelages@hotmail.com, aurinice@globo.com 2 Universidade Estadual do Rio de Janeiro, Universidade Estácio de Sá- Brasil. rfemygdio@gmail.com

3 Universidade Federal do Rio Grande do Norte-Brasil. mik@ufrnet.br

Recebido em 8 Junho 2007/Enviado para Modificação 18 Outubro 2007/Aprovado em 18 Outubro 2007

Objetivo Ao objetivo deste estudo foi analisar comparativamente o gasto calórico de universitários durante a prática da dança folclórica e da caminhada.

Material e Métodos Pesquisa descritiva aplicada em 35 universitários, voluntários, com idade variando e 19 a 34 anos, de ambos os sexos. Membros do Corpo de Dança da Universidade Estadual do Piauí, na cidade de Teresina, nas atividades de dança folclórica e caminhada. Com a balança Filizola e fita métrica Sanny, avaliouse a Massa Corporal e a Estatura e com o acelerômetro Caltrac 100/100 Plan, o gasto calórico. Para tanto, utilizaram-se os testes paramétricos $t$ de Student e correlação de Pearson, observando uma significância $p<0,05$, no sentido de comparar os resultados observados.

Resultados Os resultados demonstram que não existem diferenças significativas entre os dois grupos de universitários, segundo o tempo de prática. Tal resultado constitui um forte indicativo de que a motivação e domínio da técnica, elementos diferenciadores dos dois grupos estudados, apresentam relações de compensação do gasto calórico, justificando a ausência de distinções significativas.

Conclusão Neste sentido a dança, enquanto atividade física constitui um cenário equânime no que concerne ao gasto calórico. Ainda observaram-se correlações significativas entre os níveis de gasto calórico da dança com a caminhada, além de não serem verificadas diferenças expressivas entre as mesmas, denotando que elas se equivalem.

Palavras Chaves: Exercício aeróbico, atividade motora, saúde (fuente: DeCS, BIREME).

ABSTRACT

Objective This study was aimed at performing a comparative analysis of 
undergraduates' calorie output during folk-dancing and walking.

Materials and Methods This was a descriptive study of the calorific output of 35 volunteer students belonging to the Universidade Estadual do Piauí's Dance Company (Teresina, Brazil) ranging in age from 19 to 34, including people from both genders allocated to two groups (dancers and walkers). A Filizola balance was used for estimating body mass, a Sanny metric scale for measuring height and a Caltrac acelerometer 100/100 Plan for measuring energy output. Student ttest and Pearson's correlation were used for statistical analysis $(p<0.05$ significance level) when comparing results.

Results The results revealed no significant differences between the two undergraduate groups according to duration of activity. Such finding strongly indicates that motivation and dominating the technique (being contrasting elements in the two groups studied) compensated for calorie output, thereby explaining the absence of significant differences.

Conclusions Dancing was seen to be equal to walking as physical activities in terms of calorific output. A significant correlation was found between calorie output levels in both dancing and walking (even though no significant differences between them were verified).

Key Words: Aerobic exercise, physical fitness, health (source: $\mathrm{MeSH}, \mathrm{NLM}$ ).

\section{RESUMEN}

Objetivo El objetivo de este estudio fue analizar comparativamente el gasto calórico de una muestra de universitarios durante la práctica de un baile folclórico y durante una caminada.

Material y método Investigación descriptiva que compara el gasto calórico de 35 estudiantes universitarios voluntrios, con edades de 19 a 34 años, de ambos sexos, miembros del Cuerpo de Danza de la Universidad Estatal de Piauí, en la ciudad de Teresina. Con una balanza Filizola y una cinta métrica Sanny se evaluó la masa corporal y la estatura, y el gasto calórico con un acelerómetro Caltrac. Para ello se utilizaron pruebas paramétricas $t$ de Student y correlaciones de Pearson, con una significancia de $p<0,05$.

Resultados Los resultados demuestran que no existen diferencias significativas entre ambos grupos de universitarios desde el punto de vista del tiempo de práctica de la actividad. Tal resultado sugiere con fuerza que la motivación y el dominio de la técnica, elementos diferenciadores entre los dos grupos, presentan relaciones de compensación del gasto calórico.

Conclusiones En esta dirección la danza, como actividad física, constituye un escenario apropiado en cuanto al gasto calórico. También se observaron correlaciones significativas entre los niveles de gasto calórico en la danza y en el paseo, sin diferencias notables entre las mismas.

Palabras Claves: Gasto energético, danza, salud (fuente: DeCS, BIREME). 
REVISTADE SALUD PÚBLICA· Volumen 9 (4), Diciembre 2007

\begin{abstract}
importância da atividade física regular na prevenção de doenças e de mortes prematuras e na manutenção de uma alta qualidade de vida é, 1 hoje, reconhecida como objetivo de saúde pública, em virtude da economia direta que o país pode alcançar com o combate ao sedentarismo (1). Atualmente tem-se mais de 2 milhões de mortes atribuídas à inatividade física a cada ano no mundo inteiro(2).
\end{abstract}

Algumas alterações benéficas nos sistemas do corpo ocorrem rapidamente, como o aumento da ventilação pulmonar e a capilarização periférica, que promovem uma melhor irrigação sanguínea. Em longo prazo, os benefícios da atividade física são menos perceptíveis, porém mais intensos, como o aumento das capacidades cardíacas e pulmonares, assim como maior eficiência do sistema músculo esquelético, refletindo em um bom estado geral do organismo, bem como na diminuição da gordura corporal, ajudando a controlar a massa corporal (1).

O aumento do gasto energético através da atividade física é meio efetivo para a redução da gordura corporal (3). Por essa razão, a comunidade científica reconhece ser prudente explorar maneiras através das quais se possa aumentar o gasto energético diário, a fim de reduzir ou controlar a prevalência de obesidade (4). O gasto energético total é composto por três componentes: metabolismo de repouso, efeito térmico dos alimentos e atividade física, sendo influenciados por fatores genéticos. A atividade física é o componente mais variável e diz respeito ao gasto energético necessário à atividade músculo esquelético $(5,6)$.

Diante do exposto, percebe-se que a dança, como atividade física, pode ser utilizada efetivamente em um programa de controle de Massa Corporal e da redução dos fatores de risco de doença crônico-degenerativa, devido ao fato de contemplar os componentes aeróbicos e flexibilidade, com o diferencial de permitir que o participante sinta uma sensação de alegria e liberdade, além de possibilitar o seu relacionamento e prazer em conviver socialmente com pessoas pertencentes ou não ao mesmo grupo etário (7).

Assim, desejando contribuir para o melhor conhecimento da dança folclórica como atividade física, o objetivo deste estudo foi analisar o gasto calórico de universitários durante a prática da dança folclórica e a caminhada, uma vez que essa última constitui na preferência das indicações quando da orientação pela inserção de hábitos saudáveis, discriminados segundo os seus respectivos tempos de prática. 


\section{MATERIAL E MÉTODO}

O estudo caracterizou-se como uma pesquisa descritiva comparativa (8) do gasto calórico de dançarinos da Universidade Estadual do Piauí, em duas atividades distintas, dança folclórica e caminhada.

A pesquisa foi realizada com 34 universitários, voluntários, na faixa etária variando de 19 a 34 anos, de ambos os sexos, que participam do Corpo de Dança da Universidade Estadual do Piauí-UESPI, Campus Poeta Torquato Neto, na cidade de Teresina.

Este estudo foi desenvolvido de acordo com as Normas de Realização de Pesquisa em Seres Humanos, Resolução 196/96, do Conselho Nacional de Saúde, e avaliado pelo Comitê de Ética da Faculdade de Ciências Médicas da UESPI, pelo protocolo CEP no 097 /06 que aprovou a realização deste estudo.

Foram tomadas inicialmente medidas antropométricas dos indivíduos da pesquisa-Massa Corporal medida em Kg numa balança Filizola e a Estatura medida em centímetros, por uma fita métrica Sanny $(9,10)$.

Para a quantificação do gasto calórico na Dança (gcdança-Kcal) e na Caminhada (gccaminhada-Kcal) foi utilizado o acelerômetro Caltrac 100/100 Plan (11,12), aplicado durante uma prática de dança folclórica e de caminhada, com o tempo de duração de 1 hora cada atividade, medida através do METs (unidade de medida do gasto calórico).

A aula de dança folclórica foi composta de 5 minutos de alongamento, 5 minutos de aquecimento, 45 minutos aplicando-se elementos básicos, como locomoções (sucessões de transferências) e giros (meio giro, voltas), individualmente e aos pares, e para finalizar 5 minutos de alongamento.

A caminhada foi realizada em pista de atletismo de 400 metros, numa velocidade de 4 minutos em cada volta da pista. Essa velocidade foi encontrada através da média de velocidade desenvolvida entre 6 participantes do grupo pesquisado, dois com estaturas mais altas, dois com estaturas medianas e dois com estaturas mais baixas. Para que a caminhada tivesse o tempo correspondente da aula de dança, foram realizados 5 minutos de alongamento no início e final da caminhada. 
REVISTADE SALUD PÚBLICA· Volumen 9 (4), Diciembre 2007

O Tratamento Estatístico utilizou o software SPSS 14, licenciado no Laboratório de Biociências da Motricidade Humana da Universidade Federal do Rio Grande do Norte-Brasil, como plataforma para tabulação e análise dos dados.

O presente estudo observou dois momentos no Tratamento Estatístico, o primeiro relativo à Estatística Descritiva, no sentido de caracterizar a amostra observada segundo as variáveis verificadas. Para as variáveis de natureza paramétrica, utilizaram-se os parâmetros de média e desvio-padrão e para as de natureza não paramétricas as Distribuições de Freqüências. O segundo momento consistiu na aplicação dos testes de hipóteses, compondo a análise inferencial do Tratamento Estatístico.

Primeiramente empregou-se o teste de normalidade de Komogorov-Smirnov, no sentido de certificar-se se as variáveis estavam normalmente distribuídas. Posteriormente, o teste de hipótese $t$ de Student no modo pareado e de médias independentes, combinados ao teste de correlação de Pearson, no sentido de analisar comparativamente os valores médios dos gastos calóricos quantificados, segundo os grupos estudados. Todos os protocolos estatísticos observaram um nível de significância estatística p <0,05.

\section{RESULTADOS}

Ao aplicar-se inicialmente o teste de verificação de normalidade de KomogorovSmirnov, a Tabela 1 apresenta distribuição não diferente significativamente de uma distribuição normal em todas as variáveis Massa Corporal, Estatura, gcdança e gccaminhada.

No sentido de controlar o viés tempo de prática nos resultados quantificados de gasto calórico, a amostra foi dividida em dois grupos: Avançado (praticantes de dança com tempo de prática superior a um ano) e Iniciantes (praticantes de dança com tempo de prática inferior a um ano). As respectivas distribuições de freqüências seguem conforme a tabela a seguir:

Tabela 1. Distribuição de freqüência sexo versus tempo de prática

\begin{tabular}{lcccc}
\hline \multicolumn{1}{c}{$\begin{array}{c}\text { Grupos } \\
(n=34)\end{array}$} & \multicolumn{2}{c}{ Masculino } & \multicolumn{2}{c}{ Feminino } \\
\cline { 2 - 5 } & $n$ & $\%$ & $n$ & $\%$ \\
\hline 1(iniciante) & 1 & 3 & 13 & 38 \\
2(avançado) & 8 & 23 & 12 & 35 \\
Total & 9 & 26 & 25 & 74
\end{tabular}


Da distribuição anterior temos que, preponderantemente, os indivíduos do sexo feminino são mais freqüentes em ambos os grupos, segmentados segundo o Tempo de Prática (1-Iniciante e 2-Avançado); todavia, os grupos apresentam significativas diferenças quanto à freqüência de indivíduos do sexo masculino, sendo o Grupo 1 com 7,1\% e o Grupo 2 com 40,0\%. Neste sentido, objetivandose evitar a distorção do parâmetro sexo, para a realização das comparações inter-grupos, selecionaram-se somente as mulheres, Grupo 1 com 13 indivíduos e Grupo 2 com 12 indivíduos.

Tabela 2. Medidas das variáveis experimentais

\begin{tabular}{cccc}
\hline $\mathrm{n}=34$ & Média & D.P & Sg.p \\
\hline Massa Corporal & 58,8 & 13,5 & 0,05 \\
Estatura & 164,4 & 7,3 & 0,18 \\
godança & 275,4 & 59,1 & 0,10 \\
gocaminhada & 278,4 & 91,9 & 0,18 \\
\hline
\end{tabular}

O primeiro processo comparativo (Tabelas 2 e 3) consistiu no cruzamento pareado das variáveis relativas ao Gasto Calórico, quantificados na Dança e na Caminhada. Para tanto, utilizou-se o teste t pareado, combinado ao teste de correlação de Pearson ( $t=0,820$ e r=0,554), observando um nível de significância $\mathrm{p}<0,05$.

Tabela 3. Médias experimentais por tempo de prática

\begin{tabular}{lccc}
\hline \multicolumn{1}{c}{ Variável } & Grupo & Média & D.P \\
\hline Massa Corporal & 1(Iniciante) & 57,2 & 3,7 \\
& 2(Avançado) & 59,9 & 3,0 \\
Estatura & 1(Iniciante) & 162,4 & 1,9 \\
& 2(Avançado) & 165,7 & 1,6 \\
gcdança & 1(Iniciante) & 255,1 & 18,5 \\
& 2(Avançado) & 289,5 & 10,7 \\
gccaminhada & 1(Iniciante) & 265,4 & 26,2 \\
\hline
\end{tabular}

Dos resultados acima pode-se verificar que o grupo de indivíduos analisados apresentou níveis de gasto calórico iguais e proporcionais nas duas atividades solicitadas, dança e caminhada, não existindo, pois, diferenças significativas entre os mesmos.

Na análise comparativa dos resultados entre os grupos, considerando-se somente os indivíduos do sexo feminino, observou-se o teste t de Student para amostras independentes e um nível de significância $\mathrm{p}<0,05$. De acordo com 
REVISTADE SALUD PÚBLICA · Volumen 9 (4), Diciembre 2007

os resultados apresentados na Tabela 2, percebe-se que não foram observadas diferenças significativas entre os valores médios dos dois grupos, segmentados segundo o tempo de prática, para as variáveis Massa Corporal, Estatura, Gasto Calórico Dança e Caminhada. Tal resultado remete à discussão mais abrangente e conceitual da fisiologia do exercício para o real entendimento dos mesmos.

\section{DISCUSSÃO}

Ao combinarem-se analiticamente os parâmetros tempo de prática e as variáveis relativas ao gasto energético, partiu-se do pressuposto que quanto maior fosse o Tempo de Prática melhor seria o domínio gestual e, portanto, a otimização dos movimentos envolvidos na dança e a conseqüente minimização das variáveis relacionadas ao Gasto Energético. Componentes outros, também envolvidos no contexto da dança, estariam dentro de uma matriz de possibilidades que congregariam valências Psicológicas (motivação), Ambientais (características do meio no qual os indivíduos realizam a dança), além de todos os demais parâmetros direta ou indiretamente relacionados às interações meio e dançarino. Ressalta-se, contudo, que dentre esses parâmetros anteriormente mencionados, somente a motivação constitui fator não controlado neste estudo.

Estudos $(14,15)$ confirmam que a relação entre a intensidade de motivação e a dificuldade da tarefa é curvilínea, isto é, com uma dificuldade muito baixa ou extremamente muito alta; a motivação é mínima, com uma dificuldade média, a motivação é máxima. Tarefas fáceis produzem monotonia e saturação psíquica. Tarefas muito difíceis têm como conseqüência fracasso e frustração. Logo, os indivíduos menos experientes na dança têm muito mais a experimentar com a prática desta do que os que já têm certo tempo de execução deste tipo de movimento corporal, implicando, pois, num nível motivacional intrínseco maior nesses indivíduos comparativamente aos mais experientes $(14,15)$.

A análise acima também pode ser confirmada através de um estudo com jogadores de futebol, que mostrou uma tendência dos atletas com menos tempo de prática possuírem uma maior motivação intrínseca (16).

No âmbito da atividade física, a motivação é produto de um conjunto de variáveis sociais, ambientais e individuais, que determinam a escolha de uma modalidade física ou esportiva, e a intensidade prática dessa modalidade é que determinará o rendimento (15). 
De outra forma, se houver o entendimento que intensidade constitui uma integral sob uma curva de desempenho $\mathrm{X}$ e que quanto maior a integral, maior será a intensidade, pode-se admitir que os indivíduos com maior domínio da técnica na dança apresentarão um valor de integral maior, se comparados àqueles indivíduos com menor domínio do ato gestual, isto é, os menos experientes. Desta forma, enquanto a intensidade da Atividade Física estimada cresce no contexto dos dançarinos menos experientes, em função do aumento da motivação intrínseca, depara-se, por outro, que no universo dos dançarinos mais experientes há um aumento da intensidade da Atividade Física estimada, em virtude destes terem um maior domínio gestual e consequentemente implicar num maior valor da integral da curva de desempenho pelo tempo de execução de uma tarefa, que cresce na mesma medida em que cresce o domínio da técnica. Em contraponto, no cenário de não domínio, notam-se interrupções nas composições gestuais, que se dão sobre retas e não sobre curvas, que definem o movimento ideal.

Desta forma, pode-se entender o porquê da não interferência do tempo de prática como variável discricionária da variável dependente Gasto Calórico.Tal fato gera duas orientações. A primeira que a dança enquanto atividade física funciona como elemento normatizador quanto ao gasto calórico, isto é, uma vez que dois parâmetros diretamente relacionados ao gasto calórico, Motivação e Domínio da técnica, quando combinados, funcionam como elementos que se complementam em forma compensatória mantendo os níveis de gasto calórico constantes (não diferença significativa entre os dois grupos, novatos e experientes). A segunda orienta ao profissional da dança que, sendo níveis iguais, deve-se verificar se os indicadores de gasto calórico são satisfatórios, principalmente para o novato, no sentido de não lhe causar desconforto e, conseqüentemente, redução na sua aderência da prática desta atividade. Daí, tendo idéia do índice de gasto calórico através dos parâmetros aqui destacados, pode-se constituir um planejamento mais adequado à população alvo, com maior assertividade e maximização da aderência dos praticantes.

Os resultados denotam que o fator Tempo de Prática não constitui um parâmetro diferenciador do Gasto Energético, e que, portanto, a técnica adquirida ao longo do tempo de prática substancializa otimizações gestuais, mas não traduzidas em redução do Gasto Energético. É possível admitir que tal fato possa estar observando o viés de fatores psicológicos não controlados.

Ainda os resultados apontam que relações compensatórias se dão no processo de execução e realização do gestual da dança, devido o pressuposto 
REVISTADE SALUD PÚBLICA· Volumen 9 (4), Diciembre 2007

de que não existem diferenças no Gasto Energético entre diferentes Tempos de Prática, levando a crer que esta indiferença experimental faz da dança um ambiente integrador e normatizador, quando vista na ótica dos benefícios da Atividade Física e derivados do Gasto Calórico nela observados, não importando, pois, quando o indivíduo começou a dançar, mas sim como ele dança, quais os elementos motivacionais que o provocam, no desempenho motor que o predispõe a maior ou menor Gasto Calórico.

Conclui-se, portanto, que o gasto calórico observado na dança é igual tanto para o iniciante, como para o avançado, justificado por diferentes níveis de motivação e domínio da técnica, existindo, assim, uma correlação diretamente proporcional entre o gasto calórico observado na caminhada e na dança.

Sugere-se, assim, que em trabalhos futuros busque-se controlar fatores motivacionais e ambientais, no sentido de evitar tendenciosidade dessa natureza, como também aumentar o universo amostral que constitui sempre numa medida positiva para potencializar a relevância e significância do trabalho. Recomendase também a indicação da dança nos programas de atividade física, tratandose de uma atividade prazerosa e promotora do gasto energético •

\section{REFERÊNCIAS}

1. Pardini R, Matsudo S, Araújo T, Matsudo V, Andrade E, Braggion G, et al. Validação do Questionário Internacional de Nível de Atividade Física (IPAQ-Versão 6): Estudo Piloto em Adultos Jovens Brasileiros. Rev. Brás. Ciên. e Mov. 2001;9(3):45-51.

2. Almeida EB, Xavier GNA, Carminatti LJ, Giustina MCD. Gasto Calórico nas Atividades de Trabalho e Cotidianas dos Carteiros que Utilizam Bicicleta. Rev. Bras. Cine. Des. Hum. 2004;6(2):53-61.

3. Meirelles CM, Gomes PSC. Efeitos Agudos da Atividade Contra Resistência sobre o Gasto Enérgico: Revisando o impacto das Principais Variáveis. Rev Bras Med Esporte. 2004;10(2):122-130.

4. Carvalho T, Nóbrega CL, Lazzoli JK, Magni JRT, Rezende L, Drummond FA, at al. Posição Oficial da Sociedade Brasileira de Medicina do Esporte: Atividade Física e Saúde. Rev. Bras. Med. Esport.1996;2(4):79-81.

5. Bouchard C. Heredity and the Path to Overweight and Obesity. Med. Sci. Sports Exerc.1991;23:285-291.

6. Amorim PR; Gomes TNP. Gasto Enérgico na Atividade Física. Rio de Janeiro: Shape; 2003.

7. Strazzacappa M. A Educação e a Fábrica de Corpos: A Dança na Escola. Cadernos CEDES. 2001;21(53):1-11. 
8. Thomas JR, Nelson JK. Métodos de Pesquisa em Atividade Física. 3ed. Porto Alegre: Artmed;2002.

9. Alvarez BR, Pavan AL. Alturas e Comprimentos. in: Petroski EL. Antropometria:Técnicas e Padronizações. 2 ed. Porto Alegre:Palotti;2003. p. 59-71.

10. Samulski D. Psicologia do Esporte.Barueri: Manole; 2002.

11. Ma RO, Chow B. Validity and reliability of accelerometry and pedometryin assessing physical activity in children hith mental retardation. Med Sci Sports Exerc. 2003; 35(5), Supplement 1:223-230.

12. Rowlands AV, Thomas PWM, Eston RG, Topping R. Validation of the RT3 Triaxial Accelerometer for the Assessment of Physical Activity. Med Sci Sports Exerc. 2004;36(3):518-524.

13. Farinatti PTV.Apresentação de uma Versão em Português do Compêndio de Atividades Físicas: uma contribuição aos pesquisadores e profissionais em fisiologias do exercício. Revista Brasileira de Fisiologia do Exercício. 2003;(2):177-208.

14.Weineberg RS, Gould D. Fundamentos da Psicologia do Esporte do Exercício. 2ed. Porto Alegre: Artmed; 2001.

15. Andrade C, Keller B, Okazaki FHA, Oliveira S, Coelho RW. A Influência de Tempo de Prática na Motivação Intrínseca de Atletas de Futebol Masculino da Categoria Juvenil de Clubes Profissionais [Internet]. Disponível em www.efdeportes.com/ efd96/motivac.htm. Revista Digital. 2006;11(96):1-7.

16. Hernandez JAE, Voser RC, Lykawka MGA. Motivação no Esporte de Elite: Comparação de Categorias do Futsal e Futebol [Internet]. Disponível em www.efdeportes.com/efd77/motiv.htm. Revista Digital. 2004;10(77):1-7. 